\title{
Isolated thrombosis after COVID-19 vaccination: case series
}

\author{
Mona Al-Ahmad $^{1,2} \cdot$ Mona Al Rasheed $^{3} \cdot$ Lulwa Altourah $^{3} \cdot$ Tito Rodriguez-Bouza $^{4} \cdot$ Neveen Shalaby $^{3}$
}

Received: 26 August 2021 / Revised: 24 December 2021 / Accepted: 26 December 2021 / Published online: 6 January 2022

(c) Japanese Society of Hematology 2022

\begin{abstract}
Background Data regarding thrombosis after COVID-19 vaccination are scarce.

Methods Clinical and laboratory data were collected from all patients who developed thrombosis within 4 weeks of receiving the Pfizer or Oxford/AstraZeneca vaccine. None had a COVID-19-positive swab.

Results Seventeen patients were included, with average age of 48.8 years and equal proportion of females to males. Our data suggest that thrombosis occurred in 1 in 163,000 of all individuals who had received any dose of any type of COVID19 vaccine: six (1 in 123,000) patients after the first dose of Oxford/AstraZeneca, none after the second dose of Oxford/ AstraZeneca, four (1 in 257,000) patients after the first dose of the Pfizer vaccine, and seven $(1$ in 102,000) patients after the second dose of Pfizer vaccine. Three of 17 patients with thrombosis (17.6\%) died.

Conclusions We believe this report to be one of the earliest in the literature to address the question of whether isolated thrombosis is a possible complication of COVID-19 vaccination.
\end{abstract}

Keywords COVID-19 · Thrombosis · Vaccine

\section{Introduction}

SARS-CoV-2 was linked to an increased risk of thrombosis both during the active and post-infective period [1-3]. Several guidelines have been published addressing management of this possibly lethal complication [4, 5]. In Kuwait, the two available vaccines are Pfizer-BioNTech, and Oxford/AstraZeneca and to the time of preparing this report, over one and half million people were vaccinated [6]. However, a few serious complications were brought to attention, including several reported thrombosis cases; this raised the question of whether a valid link is present between the vaccine and the thrombosis incidences.

Mona Al-Ahmad

mona.alahmad@ku.edu.kw

1 Microbiology Department, Faculty of Medicine, Kuwait University, P.O. Box 24923, 13110 Safat, Kuwait

2 Al Rashed Allergy Centre, Ministry of Health, Kuwait, Kuwait

3 Hematology Unit, Department of Medicine, Al Adnan Hospital, Ministry of Health, Kuwait, Kuwait

4 Hospital Quirón Palmasplanas, Centro de Patología Alérgica, Balearic Islands, Spain

\section{Methods}

We conducted a single-country case-report series with all patients reported with thrombosis on the first 4 weeks after vaccination. Data were taken from January 2021 to 24 th of July 2021. For each patient, we recorded age, gender, previous medical health (PMH), baseline aspirin/anticoagulation, COVID-19 nasopharyngeal PCR swab, type of vaccine, dose of vaccine (1st or $2 \mathrm{nd}$ ), type of thrombosis, location of thrombosis, time between vaccine and onset of symptoms, nadir platelet count, D-Dimer, fibrinogen level, lupus anticoagulant, anticardiolipin (ACL), beta-2-glycoprotein (B2GP), thrombophilia screening, heparin-induced thrombocytopenia and thrombosis (HITT) ELISA assay, treatment outcome, and additional description of the case.

\section{Results}

From January 2021 till 24th July 2021, a total of 1,745,713 patients had received Pfizer vaccine in Kuwait; of these, $1,029,417$ had received the first dose only and 716,296 had received both doses of the vaccine. In contrast, a total of $1,025,715$ had received Oxford/AstraZeneca vaccine. Of these, 736,123 had received the first dose only and 289,592 
had received both doses of the vaccine. The first case of thrombosis was reported on the 22nd February 2021.

In this article, we are reporting a total number of 17 patients who had developed thrombosis after receiving COVID-19 vaccination which represents 1 in 163,000 of all individuals who had received any type or dose of the COVID-19 vaccine. Of these, 6 patients had developed thrombosis after the first dose of Oxford/AstraZeneca vaccine which represents 1 in 123,000 among all individuals who had received the first dose of Oxford/AstraZeneca vaccine in Kuwait. We did not encounter any case of thrombosis after the second dose of Oxford/AstraZeneca vaccine (Table 1).

Of the patient who developed thrombosis after Pfizer vaccine, 7 out of 11 developed the thrombosis after the second dose of the vaccine which represents 1 in 102,000 of all individuals who received the second dose of Pfizer vaccine. Four out of eleven developed thrombosis after the first dose of the Pfizer vaccine which represents 1 in 257,000 of all individuals who had received the first dose only of the Pfizer vaccine (Table 1).

Clinical characteristics, laboratory results, and management of cases are summarized in Table 1. Equal number of females to males had developed thrombosis in patient who received Oxford/AstraZeneca vaccine, in contrast to, 5 out of 11 females who had developed thrombosis in patient who had received Pfizer vaccine. Regarding previous management, only one patient was on aspirin and none were on long-term anticoagulation. Ten patients had venous thrombosis [Deep vein thrombosis (DVT)/Pulmonary embolism (PE) and two patients with cerebral venous thrombosis (CVT) (59\%)] and seven out of seventeen patients had developed arterial thrombosis [myocardial infarct (MI), cerebrovascular accident (CVA), Peripheral vascular disease (PVD) (41\%)]. There was one patient who developed both arterial and venous thrombosis. In patients who received Pfizer vaccine, 9 out of 11 (82\%) developed venous thrombosis and 2 out of $11(18 \%)$ developed arterial thrombosis. In patients who received Oxford/AstraZeneca vaccine, 2 out of $6(33.3 \%)$ developed venous thrombosis compared to 4 out of $6(66.6 \%)$ who developed arterial thrombosis. The mean time between vaccine exposure and the thrombosis is 13 days with a median of 14 days and a range of 25 days (1-26 days.). All patients had COVID-19 nasopharyngeal PCR negative swab. Two patients only had thrombosis with thrombocytopenia, one was after the first dose of the Oxford/ AstraZeneca vaccination and the other one was after 2 nd dose of Pfizer vaccine, which may represent the first case of Vaccine-Induced Immune Thrombotic Thrombocytopenia (VITT) after Pfizer vaccine. In the first case, hematology service was not consulted and HITT assay was not sent, while in the second case, HITT assay was sent, but the reagent was not available. There was one patient who developed massive
CVA, and she had very high D-Dimer but no thrombocytopenia (complete blood cell (CBC) analysis on admission was normal, with no subsequent $\mathrm{CBC}$ ). This case can be considered as suspected cases of VITT. HITT assay was sent, but the reagent was not available. Seven patients had thrombophilia screening done. Four of those had lupus anticoagulant positive (repeated twice 3 months apart) with negative ACL and B2GP, and one had borderline low protein S level, while the rest had negative thrombophilia screening. Three out of the seventeen patient had passed away. All the three-patient had arterial rather than venous thrombosis. Two patients had received the first dose of Oxford/AstraZeneca vaccine and one had received the second dose of Pfizer vaccine. Two of those patients had very high D-Dimer in addition to thrombocytopenia and one had very high D-Dimer without thrombocytopenia which can potentially represent suspected cases of VITT. Unfortunately, HITT assays were not sent or not available for any of these patients to confirm possibility of VITT.

\section{Discussion}

The emergency release of the Pfizer vaccine was in December 11th 2020 and of Oxford/AstraZeneca was on March 17th 2021 [7, 8]. Thrombosis has not been described as a possible vaccine complication in initial clinical trials [7,9]; moreover, there has not been any case reports or case series in the literature linking thrombosis to vaccination except those describing VITT [10-13]. A safety report published by health product regulatory authority stated that a few cases developed thrombosis; however, those patients already had risk factors for such complication [14]. Another paper collecting data from Vigibase, a Sweden databank used to gather information about adverse drug reactions, stated that thrombosis has been reported post-vaccination; nevertheless, a comparison between vaccinated and unvaccinated individuals is needed to determine a true association. In this article, both arterial and venous thrombosis has been reported, including unusual site thrombosis, such as cerebral vein thrombosis, similar to our reported cases. People vaccinated with mRNA-based vaccine (e.g., Pfizer/BioNTech) showed more venous than arterial thrombosis compared to those vaccinated by Oxford/AstraZeneca who had equal number of arterial and venous thrombosis. Thrombocytopenia associated with positive HITT assay, disseminated intravascular coagulation, and positive antiphospholipid antibodies were also reported in patients who received COVID-19 vaccines [15].

A recent publication [16] showed an association between the first dose of Oxford/AstraZeneca vaccine and arterial thrombosis. No similar association was found between Oxford/AstraZeneca vaccine and venous thrombosis nor Pfizer vaccine with arterial or venous thrombosis. In our 
Table 1 Clinical characteristics, laboratory results, and management of cases

\begin{tabular}{|c|c|c|c|}
\hline Vaccine & $\begin{array}{l}\text { Pfizer } \\
1 \text { st dose }\end{array}$ & $\begin{array}{l}\text { Pfizer } \\
\text { 2nd dose }\end{array}$ & $\begin{array}{l}\text { Oxford/AstraZeneca } \\
\text { 1st dose }\end{array}$ \\
\hline Age, average (SD) & $39.5(18.4)$ & $58.9(15.8)$ & $43.3(8.3)$ \\
\hline \multicolumn{4}{|l|}{ Gender } \\
\hline Female & 2 & 3 & 3 \\
\hline Male & 2 & 4 & 3 \\
\hline \multicolumn{4}{|l|}{ PMH } \\
\hline Healthy & 2 & 4 & 5 \\
\hline $\mathrm{DVT} / \mathrm{PE}$ & 1 & 0 & 0 \\
\hline $\mathrm{DM}+$ smoker & 0 & 0 & 1 \\
\hline $\mathrm{DM}+\mathrm{HTA}$ & 1 & 3 & 0 \\
\hline \multicolumn{4}{|c|}{ Thrombotic events in relation to individuals received vaccine } \\
\hline Total: $1 / 163,000$ & $1 / 257,000$ & $1 / 102,000$ & $1 / 123,000$ \\
\hline \multicolumn{4}{|l|}{ Type of thrombosis } \\
\hline Venous & 3 & 3 & 2 \\
\hline Arterial & 1 & 3 & 4 \\
\hline Both & 0 & 1 & 0 \\
\hline \multicolumn{4}{|l|}{ Location of thrombosis } \\
\hline PE & 1 & 1 & 0 \\
\hline DVT & 1 & 2 & 0 \\
\hline Cardiac & 0 & 1 & 1 \\
\hline CVA & 0 & 1 & 3 \\
\hline CVT & 0 & 1 & 1 \\
\hline Limbs & 1 & 0 & 0 \\
\hline $\mathrm{DVT}+\mathrm{PE}$ & 1 & 0 & 1 \\
\hline $\mathrm{DVT}+\mathrm{CVA}$ & 0 & 1 & 0 \\
\hline $\begin{array}{l}\text { Average days from } \\
\text { vaccine to symptoms (SD) }\end{array}$ & $13(5.7)$ & $14.3(8.5)$ & $13.5(5.8)$ \\
\hline Nadir platelet $\left(\times 10^{9} / \mathrm{L}\right)$ & $262(85.1)$ & $262(104.9)$ & $280.8(130.5)$ \\
\hline D-Dimer (ng/ml DDU) & $1542(1236.6)$ & $\begin{array}{l}706.5 \\
(598)\end{array}$ & $\begin{array}{l}3125 \\
(3283.9)\end{array}$ \\
\hline Thrombocytopenia or very high D-Dimer & 0 & 1 & 2 \\
\hline Fibrinogen $(\mathrm{g} / \mathrm{l})$ & $2.6(0.5)$ & $2.9(1.5)$ & $2.7(0.7)$ \\
\hline \multicolumn{4}{|l|}{ Lupus anticoagulant $^{\mathrm{a}}$} \\
\hline Positive & $3 / 3(100 \%)$ & $1 / 4(25 \%)$ & n.d \\
\hline \multicolumn{4}{|l|}{$\mathrm{ACL}^{\mathrm{a}}$} \\
\hline Positive & $0 / 3(0 \%)$ & $0 / 4(0 \%)$ & n.d \\
\hline \multicolumn{4}{|l|}{$\mathrm{B} 2 \mathrm{GP}^{\mathrm{a}}$} \\
\hline Positive & $0 / 3(0 \%)$ & $0 / 4(0 \%)$ & n.d \\
\hline \multicolumn{4}{|l|}{ Thrombophilia screen ${ }^{\mathrm{a}}$} \\
\hline Positive & $0 / 3(0 \%)$ & $0 / 2(50 \%)$ & n.d \\
\hline \multicolumn{4}{|l|}{ HIT ELISA assay ${ }^{a}$} \\
\hline Positive & n.d & n.d & n.d \\
\hline \multicolumn{4}{|l|}{ Treatment } \\
\hline Rivoraxaban & 0 & 1 & 1 \\
\hline LMWH+ warfarin & 3 & 2 & 1 \\
\hline Heparin + rivaroxaban & 1 & 1 & 0 \\
\hline LMW + rivaroxaban & 0 & 1 & 0 \\
\hline $\mathrm{ASA}+$ rosuvastatin & 0 & 0 & 2 \\
\hline ASA + heparin + bivalrudin + IVIG & 0 & 1 & 0 \\
\hline None/not available & 0 & 1 & 2 \\
\hline \multicolumn{4}{|l|}{ Outcome } \\
\hline Stable & 4 & 6 & 4 \\
\hline Passed away & 0 & 1 & 2 \\
\hline
\end{tabular}

$D V T$ deep vein thrombosis, $P E$ pulmonary embolism, $U L$ upper limb, $C V A$ cerebrovascular accident, $C V T$ cerebral venous thrombosis. ACL anticardiolipin antibody, B2GP beta2 glycoprotein, HITT assay heparin- 
Table 1 (continued)

induced thrombotic thrombocytopenia assay, $L M W H$ low-molecular-weight heparin, IVIG intravenous gammaglobulin, n.d. Not done

${ }^{a}$ Numbers are expressed as the positive results among those who made the test

case series, all patients who developed thrombosis after Oxford/AstraZeneca vaccine did so after the first dose of the vaccine which was consistent with findings from the previous study.

In conclusion, isolated thrombosis was not reported as one of the vaccines adverse events in the earliest vaccine randomized controlled trials [9, 17]. However, several other adverse events were also reported post emergency release of the vaccine such as VITT [10-13]. A true link between our reported isolated thrombosis cases and the vaccine is yet to be established by a large cohort study. If a true link was to be established, the mechanism by which the vaccine plays a role in causing thrombosis is unclear. Whether it affects any aspect of Virchow's triad: hypercoagulability, stasis, or vascular endothelia damage or has a new undiscovered mechanism is yet to be proven [18]. However, since thrombosis is possibly fatal, reporting such incidences could raise awareness and start an investigation searching for true link between the two events. We believe this report to be one of the earliest in the literature to address the question of whether isolated thrombosis is a possible complication of COVID-19 vaccine, the specific type of the vaccine causing the thrombosis and whether thrombosis occurs after the first or the second dose of the vaccine.

Supplementary Information The online version contains supplementary material available at https://doi.org/10.1007/s12185-021-03285-6.

\begin{abstract}
Author's contribution MA and MA initiated and coordinated the development of the paper, and worked on data collection, analysis, and writing up the paper. MA, MA, LA, TR, and NS analyzed and interpreted the results and helped in writing up introduction. All authors read and approved the final manuscript.
\end{abstract}

Data availability The datasets used and/or analyzed during the current study are available from the corresponding author on reasonable request.

\section{Declarations}

Conflict of interest The authors have no conflicts of interest to declare.

\section{References}

1. Chi G, Lee JJ, Jamil A, Gunnam V, Najafi H, Memar Montazerin $\mathrm{S}$, et al. Venous thromboembolism among hospitalized patients with COVID-19 undergoing thromboprophylaxis: a systematic review and meta-analysis. J Clin Med. 2020;9(8):2489.

2. Criel M, Falter M, Jaeken J, Van Kerrebroeck M, Lefere I, Meylaerts L, et al. Venous thromboembolism in SARS-CoV-2 patients: only a problem in ventilated ICU patients, or is there more to it? Eur Respir J. 2020;56(1):2001201. https://doi.org/ 10.1183/13993003.01201-2020. Accessed 30 Jul 2020.

3. Mohamed MFH, Al-Shokri SD, Shunnar KM, Mohamed SF, Najim MS, Ibrahim SI, et al. Prevalence of venous thromboembolism in critically Ill COVID-19 patients: systematic review and meta-analysis. Front Cardiovasc Med. 2020;7:598846.

4. Marietta M, Ageno W, Artoni A, De Candia E, Gresele P, Marchetti M, et al. COVID-19 and haemostasis: a position paper from Italian Society on thrombosis and haemostasis (SISET). Blood Transfus. 2020;18(3):167-9.

5. Spyropoulos AC, Levy JH, Ageno W, Connors JM, Hunt BJ, Iba $\mathrm{T}$, et al. Scientific and standardization committee communication: clinical guidance on the diagnosis, prevention, and treatment of venous thromboembolism in hospitalized patients with COVID-19. J Thromb Haemost. 2020;18(8):1859-65.

6. https://covidvax.live/location/kwt. Accessed 25 Aug 2021.

7. https://www.astrazeneca.com/media-centre/press-releases/2020/ astrazenecas-covid-19-vaccine-authorised-in-uk.html. Accessed 25 Aug 2021.

8. https://www.fda.gov/news-events/press-announcements/fdatakes-key-action-fight-against-covid-19-issuing-emergencyuse-authorization-first-covid-19. Accessed 25 Aug 2021.

9. Polack FP, Thomas SJ, Kitchin N, Absalon J, Gurtman A, Lockhart S, et al. Safety and efficacy of the BNT162b2 mRNA Covid-19 vaccine. N Engl J Med. 2020;383(27):2603-15.

10. Karnam A, Lacroix-Desmazes S, Kaveri SV, Bayry J. Vaccineinduced prothrombotic immune thrombocytopenia (VIPIT): consider IVIG batch in the treatment. J Thromb Haemost. 2021;19(7):1838-9.

11. Mehta PR, Apap Mangion S, Benger M, Stanton BR, Czuprynska J, Arya R, et al. Cerebral venous sinus thrombosis and thrombocytopenia after COVID-19 vaccination - a report of two UK cases. Brain Behav Immun. 2021;95:514-7.

12. Parums DV. Editorial: SARS-CoV-2 mRNA vaccines and the possible mechanism of vaccine-induced immune thrombotic thrombocytopenia (VITT). Med Sci Monit. 2021;27:e932899.

13. Porres-Aguilar M, Lazo-Langner A, Panduro A, Uribe M. COVID-19 vaccine-induced immune thrombotic thrombocytopenia: an emerging cause of splanchnic vein thrombosis. Ann Hepatol. 2021;23:100356.

14. https://www.hpra.ie/docs/default-source/default-document-libra ry/safety-update-covid-19-vaccines-overview-of-national-repor ting-experience-(20052021).pdf?sfvrsn=4. Accessed 25 Aug 2021.

15. Smadja DM, Yue QY, Chocron R, Sanchez O, Lillo-Le LA. Vaccination against COVID-19: insight from arterial and venous thrombosis occurrence using data from VigiBase. Eur Respir J. 2021;58(1):2100956. https://doi.org/10.1183/13993003.009562021. Accessed 1 Jul 2021.

16. Simpson CR, Shi T, Vasileiou E, Katikireddi SV, Kerr S, Moore E, et al. First-dose ChAdOx1 and BNT162b2 COVID-19 vaccines and thrombocytopenic, thromboembolic and hemorrhagic events in Scotland. Nat Med. 2021;27(7):1290-7.

17. AstraZeneca AZD. A Phase III Randomized, Double-blind, Placebo-controlled Multicenter Study in Adults to Determine the Safety, Efficacy, and Immunogenicity of AZD1222, a Nonreplicating ChAdOx1 Vector Vaccine, for the Prevention of COVID-19. 2020. https://clinicaltrials.gov/ct2/show/NCT04 
516746 ? term $=$ NCT04516746\&draw $=2 \&$ rank $=1$. Accessed Feb 2021.

18. Frick G, Frick U. Recent aspects of thrombogenesis and thrombosis prevention. Z Gesamte Inn Med. 1975;30(17):561-7.
Publisher's Note Springer Nature remains neutral with regard to jurisdictional claims in published maps and institutional affiliations. 